\title{
Marketing Social e a Promoção dos Direitos da Mulher: um Estudo de Caso
}

Aline Bezerra Franchini ${ }^{1}$; Bruno Diniz Gomes da Silva ${ }^{2}$; Maria Gabriela Jandiroba Silva ${ }^{3}$

Resumo: Este estudo objetivou analisar, sob a ótica do marketing social, a contribuição das ações promovidas pelo Conselho Municipal dos Direitos da Mulher - CMDM na missão de garantir os direitos das mulheres do município de Paulo Afonso - BA. O estudo possui natureza exploratória e descritiva e a estratégia de pesquisa foi o estudo de caso. Os dados foram coletados por meio de pesquisa documental e entrevistas; e analisados de forma qualitativa. Constatou-se que o Conselho Municipal dos Direitos da Mulher tem papel basilar na implementação da política pública municipal e, sobretudo, na garantia dos direitos e consolidação da rede de atendimento às mulheres pauloafonsinas vítimas de violência.

Descritores: Marketing Social; Promoção dos Direitos; Garantia dos Diretos das Mulheres

\section{Social Marketing and the Promotion Women's Rights: a Study Case}

\begin{abstract}
This study aimed to analyze, from the social marketing standpoint, the contribution of the actions promoted by the Municipal Council for Women's Rights (MCWR) with the objective of guaranteeing the rights of women in the municipality of Paulo Afonso - BA. The study method was exploratory and descriptive and the research strategy was the study case. The data were collected through documentary research and interviews; and analyzed qualitatively. It was found that the Municipal Council for Women's Rights has a fundamental role in the implementation of municipal public policies and, above all, in guaranteeing the rights and consolidation of the network of assistance to pauloafonsinas women victims of violence.
\end{abstract}

Descriptors: Social Marketing; Promotion of Rights; Guarantee Women's Rights

\section{Introdução}

A violência é um problema que devasta a sociedade desde a sua origem. Um dos tipos que vem crescendo desenfreadamente é a violência contra a mulher. Esta se revela uma constante arraigada e de complexa contenda, por diversos entraves que dificultavam a obtenção

\footnotetext{
${ }^{1}$ Especialização em Recursos Humanos com ênfase em Gestão de pessoas: Faculdade São Luís de França, 2014. Especialização em Gestão Pública (em curso); Universidade Federal do Vale do São Francisco - UNIVASF. Contato: franchini_872@ hotmail.com;

${ }^{2}$ Graduação em Serviços Sociais; Universidade Luterana do Brasil - ULBRA, 2013. Especialização em Gestão Pública (em curso).

Universidade Federal do Vale do São Francisco - UNIVASF. Contato: brunodg25@ hotmail.com;

${ }^{3}$ Mestre em Administração; docente da Universidade Federal do Vale do São Francisco - UNIVASF. Contato: gabriela.jandiroba@univasf.edu.br 
de ajuda, entre eles o receio de uma reação ainda mais agressiva do companheiro; vergonha por parte das vítimas; dependência (financeira e/ou afetiva) da vítima em relação ao agressor, dentre outras.

O instrumento que pode auxiliar no enfrentamento deste problema é a implementação, de modo eficaz, de políticas públicas que garantam a efetivação dos direitos das mulheres. De acordo com a Secretaria de Políticas para as Mulheres (2018), "políticas públicas se constituem em uma das formas de interação e de diálogo entre o Estado e a sociedade civil, por meio da transformação de diretrizes e princípios norteadores em ações, regras e procedimentos que (re) constroem a realidade".

Considerando estudo realizado pelo Portal Brasil (2016) abaixo estão relacionadas algumas formas que são consideradas violência doméstica no Brasil:

1: Humilhar, xingar e diminuir a autoestima - Agressões como humilhação, desvalorização moral ou deboche público em relação a mulher constam como tipos de violência emocional.

2: Tirar a liberdade de crença - Um homem não pode restringir a ação, a decisão ou a crença de uma mulher. Isso também é considerado como uma forma de violência psicológica.

3: Fazer a mulher achar que está ficando louca - Há inclusive um nome para isso: o gaslighting. Uma forma de abuso mental que consiste em distorcer os fatos e omitir situações para deixar a vítima em dúvida sobre a sua memória e sanidade.

4: Controlar e oprimir a mulher - Aqui o que conta é o comportamento obsessivo do homem sobre a mulher, como querer controlar o que ela faz, não deixá-la sair, isolar sua família e amigos ou procurar mensagens no celular ou e-mail.

5: Expor a vida íntima - Falar sobre a vida do casal para outros é considerado uma forma de violência moral, como por exemplo vazar fotos íntimas nas redes sociais como forma de vingança.

6: Atirar objetos, sacudir e apertar os braços - Nem toda violência física é o espancamento. São considerados também como abuso físico a tentativa de arremessar objetos, com a intenção de machucar, sacudir e segurar com força uma mulher.

7: Forçar atos sexuais desconfortáveis - Não é só forçar o sexo que consta como violência sexual. Obrigar a mulher a fazer atos sexuais que causam desconforto ou repulsa, como a realização de fetiches, também é violência. 
8: Impedir a mulher de prevenir a gravidez ou obrigá-la a abortar - $\mathrm{O}$ ato de impedir uma mulher de usar métodos contraceptivos, como a pílula do dia seguinte ou o anticoncepcional, é considerado uma prática da violência sexual. Da mesma forma, obrigar uma mulher a abortar também é outra forma de abuso.

9: Controlar o dinheiro ou reter documentos - Se o homem tenta controlar, guardar ou tirar o dinheiro de uma mulher contra a sua vontade, assim como guardar documentos pessoais da mulher, isso é considerado uma forma de violência patrimonial.

10: Quebrar objetos da mulher - Outra forma de violência ao patrimônio da mulher é causar danos de propósito a objetos dela, ou objetos que ela goste.

Em 07 de agosto de 2006 foi sancionada a Lei Federal $n^{\circ} 11.340$, popularmente conhecida como Lei Maria da Penha, que alterou o Código Penal possibilitando que agressores de mulheres em esfera doméstica ou familiar sejam presos em flagrante ou tenham sua prisão preventiva decretada. A legislação aumenta o período de detenção e prevê medidas que vão desde a retirada do agressor do domicílio à proibição de sua aproximação da vítima.

A Política Nacional de Enfrentamento à Violência contra as Mulheres foi elaborada pela Secretaria Especial de Políticas para as Mulheres (SPM) com o objetivo de explicitar os fundamentos conceituais e políticos do enfrentamento à questão e as políticas públicas que têm sido formuladas e executadas para a prevenção e combate à violência contra as mulheres, assim como para a assistência às mulheres em situação de violência.

Considerando as dificuldades enfrentadas no combate a problemas sociais (como a violência contra a mulher), sobretudo, em avaliar quais as causas e consequências destes, o marketing social surge como ferramenta estratégica para auxiliar nestes anseios e necessidades mais densas da sociedade.

O termo "marketing social” foi empregado pela primeira vez apenas em 1971 no artigo "Social marketing: An Approach to Planned Social Change", publicado no Journal of Marketing, de autoria de Kotler e Zaltman (KOTLER; ZALTMAN, 1971). Neste artigo, os referidos autores defenderam a aplicabilidade de conceitos de marketing para a promoção de causas sociais e definiram marketing social como sendo "a criação, implementação e controle de programas calculados para influenciar a aceitação de ideias sociais [grifo nosso] e envolvendo considerações de planejamento, precificação, comunicação, distribuição de produtos e pesquisa de marketing” (apud Silva, 2016 p.26). 
O conceito de Marketing Social para Kotler e Roberto (1992, p. 25) após três décadas ainda tem a mesma definição ou seja : "[...] uma tecnologia de administração da mudança social, associada ao projeto, à implantação e ao controle de programas voltados para o aumento da disposição de aceitação de uma ideia ou prática social em um ou mais grupos de adotante escolhidos como alvo".

O marketing social é a aplicação dos princípios e técnicas de marketing para criar, comunicar e entregar valor, com o objetivo de influenciar o comportamento do público-alvo em seu benefício e de toda a sociedade (LEE e KOTLER, 2009, p. 51).

Distintivamente de um empreendimento habitual, as organizações sociais necessitam comercializar um motivo e/ou uma causa. E para isso, seu usuário precisa se identificar com a causa. O maior desafio enfrentado é gerar esta percepção de necessidade em relação à causa defendida e transformar esta em um plano eficaz para alcançar os resultados almejados.

Marketing Social pode ser considerado a modalidade de ação mercadológica institucional que tem como objetivo principal atenuar ou eliminar os problemas sociais, as carências da sociedade relacionadas principalmente às questões de higiene e saúde pública, de trabalho, educação, habitação, transportes e nutrição. (VAZ, 1995, p. 280).

O Marketing social pode ser aplicado a uma pluralidade de questões sociais, compreendendo alfabetização, direitos de minorias, controle da obesidade, prevenção de abuso contra crianças, controle de armas, perigo dos fogos de artifícios, prevenção de violência contra a mulher e outros (BOONE \& KURTZ, 1998).

De acordo com Fox e Kotler (1980), as ascendências do marketing social estão na abordagem informacional, na forma de propaganda social. À medida que esforços são agregados com a realização de pesquisa de marketing, uso de incentivos, dentre outros aspectos dessa natureza, fica claro que o marketing social em si “envolve todos os 4 Ps, não apenas um". O Composto de Marketing ou Marketing Mix é um conjunto de variáveis controláveis que influenciam a forma com que os usuários respondem ao programa ou mercado.

Na percepção de Kotler (2003, p. 151), o mix de marketing "descreve o conjunto de ferramentas à disposição da gerência para influenciar as vendas". Usualmente é versada como 4 P's: Produto, Preço, Praça e Promoção (product, price, place e promotion). Se estudados com uma lógica menos mercantil, focado no entendimento de ensejos públicos sociais e em mais dois elementos do composto mercadológico social (o público-adotante e as pessoas), tais sugestões tendem a ser inovadoras (FONTES, 2001). 
No que tange ao composto de marketing social, autores como Kotler e Roberto (1992) e Fontes (2001) concordam com a adoção de mais dois "P's" (público-adotante e as pessoas), aos habituais 4 P's do Marketing convencional, visando transformações sociais, que apenas ocorrem a partir dos próprios usuários (adotantes).

A utilização dos 6Ps (público-adotante, pessoas, produto, preço, promoção e praça) pode ser considerada uma importante ferramenta de otimização de ações e recursos do governo, da sociedade civil e das empresas. $\mathrm{O}$ mix de marketing social pode ser utilizado nas mais diferentes causas sociais, vinculadas a áreas como: saúde, desenvolvimento e justiça social, defesa de direitos, comportamento ético-político, entre outros. Os 6Ps são qualificados por Kotler e Roberto (1989) e descritos por Fontes (2001), como maneira de realizar atividades no meio social afim de promover o desenvolvimento deste, conforme quadro 01 abaixo:

Quadro 01 - Mix de Marketing Social

\begin{tabular}{|c|l|}
\hline $\begin{array}{c}\text { Marketing Mix } \\
\text { Social }\end{array}$ & \multicolumn{1}{|c|}{ Características } \\
\hline Produto & $\begin{array}{l}\text { Ideias e comportamentos que promovam mudanças. Tecnologia } \\
\text { social }\end{array}$ \\
\hline Preço & Monetário ou não monetário (engajamento social) \\
\hline Praça & $\begin{array}{l}\text { Oferta a demanda, programa social ao grupo adotante escolhido com } \\
\text { alvo }\end{array}$ \\
\hline Promoção & Estratégias para acréscimo dos incentivos ao público- adotante \\
\hline Público Adotante & $\begin{array}{l}\text { Grupo de pessoas escolhidas como alvo, que possui seu próprio } \\
\text { conjunto de crenças, valores e atitudes }\end{array}$ \\
\hline Pessoal & Conjunto de pessoas capacitadas para a gestão de um projeto social. \\
\hline
\end{tabular}

Fonte: Adaptado de Kiel e Boeing da Silveira (2007, p.72)

Considerando o problema social da violência contra a mulher e a necessidade de assegurar à população feminina o pleno exercício de sua cidadania, com melhoria das condições de vida e a eliminação de todas as formas de discriminação e violência contra elas por meio de modificações estratégicas de comportamentos sociais, no dia 08 de março de 2007, através de um projeto de iniciativa popular, foi sancionada a Lei $n^{\circ}$ 1062/2007 que instituiu o Conselho Municipal dos Direitos da Mulher - CMDM do município de Paulo Afonso - Bahia. 
O CMDM é um órgão colegiado com caráter deliberativo, fiscalizador, propositivo e consultivo, que auxilia e acompanha a implementação das Políticas Públicas e Programas, sob a ótica de Gênero, em todas as esferas da Administração do Município, além de cooperar para garantir a igualdade de direitos e oportunidades entre homens e mulheres.

Então, baseado neste contexto investigou-se: Qual contribuição das ações promovidas pelo CMDM em sua missão de garantir os direitos das Mulheres de Paulo Afonso - BA? Há alguma cooperação entre órgãos que também trabalham com a promoção dos direitos da Mulher? Nesse sentido, o objetivo deste artigo foi analisar, sob a ótica do Marketing Social, a contribuição das ações promovidas pelo Conselho Municipal dos Direitos da Mulher - CMDM em sua missão de garantir os direitos das Mulheres de Paulo Afonso - BA.

\section{Métodos}

Para o alcance dos objetivos deste estudo, foi realizada uma pesquisa de natureza exploratória e descritiva. A estratégia de pesquisa foi o estudo de caso e os dados foram analisados de forma qualitativa.

No que tange a pesquisa exploratória e descritiva, Lakatos e Marconi (2001,p. 188) explicam:

São estudos exploratórios que têm por objetivo descrever completamente determinado fenômeno. [...] Podem ser encontradas tanto descrições quantitativas e/ou qualitativas quanto acumulação de informações detalhadas como as obtidas por intermédio da observação participante.

A estratégia de pesquisa utilizada foi o estudo de caso, onde Yin (2001, p.33) assegura que este "compreende um método que abrange tudo - com a lógica de planejamento incorporando abordagens específicas à coleta de dados e à análise de dados”.

Inicialmente foi realizada pesquisa documental direta para obtenção de maiores informações sobre o Conselho Municipal dos Direitos da Mulher-CMDM. Lakatos e Marconi (2001, p.186) afirmam que "constitui-se, em geral, no levantamento de dados no próprio local onde os fenômenos ocorrem". 
Posteriormente, a fim de coletar mais informações, houve a necessidade de realização de pesquisa com as principais responsáveis pela rede de proteção a mulher do município de Paulo Afonso - BA: presidente do Conselho Municipal dos Direitos da Mulher - CMDM, a delegada titular da Delegacia Especializada no Atendimento à Mulher - DEAM de Paulo Afonso - BA, a delegada titular da $18^{\mathrm{a}}$ Coordenadoria Regional de Polícia do Interior COORPIN, a delegada titular da $17^{\text {a }}$ Coordenadoria Regional de Polícia do Interior COORPIN e a Secretária Municipal de Desenvolvimento Social.

Os dados foram coletados através da aplicação de questionário, onde de acordo com Lakatos e Marconi (2001, p. 201) "é um instrumento de coleta de dados, constituída por uma série ordenada de perguntas, que devem ser respondidas por escrito e sem a presença do entrevistador".

Considerando que as entrevistadas exercem funções relevantes para a segurança e o desenvolvimento social, os questionários foram enviados, através de e-mail, no dia 14 de março de 2018 e respondidos até o dia 21 de março de 2018, visto que não há necessidade de tabulação de dados. As informações coletadas foram analisadas de forma qualitativa onde Gil (2002) considera "que há uma relação dinâmica entre o mundo real e o subjetivo, isto é, um vínculo indissociável entre o objetivo e a subjetividade do sujeito que não podem ser traduzidos em números. [...] Não requer o uso de métodos e técnicas estatísticas".

\section{Resultados e Discussão}

Foi observado que mediante iniciativa e mobilização do Conselho Municipal dos Direitos da Mulher - CMDM em parceria com a Secretaria Municipal de Desenvolvimento Social, Governo do Estado da Bahia e Secretaria de Políticas para as Mulheres do Governo Federal foi implantada uma unidade da Delegacia Especializada no Atendimento a Mulher em Paulo Afonso - BA (uma das primeiras cidades no Estado da Bahia a receber uma DEAM).

As DEAMs são unidades especializadas da Polícia Civil, que realizam ações de prevenção, proteção e investigação dos crimes de violência doméstica e violência sexual contra as mulheres, entre outros. Entre as ações, cabe citar: registro de Boletim de Ocorrência, solicitação ao juiz das medidas protetivas de urgência nos casos de violência doméstica e 
familiar contra as mulheres, realização da investigação dos crimes (SECRETARIA DE POLÍTICAS PARA AS MULHERES, 2017).

A Delegacia Especializada no Atendimento à Mulher - DEAM foi implantada em Paulo Afonso - BA sob a titularidade da delegada Mirela Santana. “A DEAM, composta por uma equipe multidisciplinar, passou junto ao Conselho Municipal dos Direitos da Mulher a acolher as mulheres vítimas de violência. Enquanto o CMDM recebia as denúncias e tomava providências junto aos poderes públicos para implantar uma rede de atenção que pudesse cuidar e restabelecer as vítimas de violência, a DEAM lavrava os procedimentos policias e fazia as prisões", afirmou Mirela Santana.

Atualmente titular da $18^{\text {a }}$ Coordenadoria Regional de Polícia do Interior - COORPIN, Mirela Santana ressaltou que, entre outras, uma grande conquista do CMDM em parceria com a DEAM foi à inauguração de uma ala feminina no Conjunto Penal de Paulo Afonso - BA tendo como benefícios, entre muitos, a transferência de mulheres em situação de prisão da delegacia territorial e construção de uma enfermaria exclusivamente feminina para atendimento humanizado das detentas. Outra ação foi a conquista de verba federal para construção do Centro de Referência da Mulher - CRM - espaço que presta acolhida, acompanhamento psicossocial e orientação jurídica às mulheres em situação de violência doméstica, sexual, patrimonial, moral, física, psicológica; além do tráfico de mulheres (BRASIL, 2018)

A delegada Lígia Nunes de Sá teve sua primeira atuação no ano de 2011 quando substituiu a titular Mirela Santana em sua licença maternidade. No ano de 2012 a mesma ficou como adjunta e em 2014 foi nomeada delegada titular da DEAM, prestando serviços até o ano de 2017 quando foi nomeada Delegada Regional da $17^{\circ}$ Coordenadoria Regional de Polícia do Interior-COORPIN.

O Conselho Municipal dos Direitos da Mulher - CMDM sempre atuou de forma marcante na defesa dos direitos das mulheres, exercendo papel basilar nas questões de gênero e na composição e alinhamento da Rede de Proteção. “Atuou em movimentos sociais em prol da instalação da DEAM e apoia a Unidade Policial em suas mais variadas demandas no combate a violência contra Mulher" afirmou Ligia Nunes.

Iniciando sua gestão em 19 de março de 2017, a atual delegada titular da DEAM Juliana Fontes afirmou que o CMDM auxilia, sobretudo, no acolhimento, tratando de forma humanizada e contribuindo para que as vítimas se sintam confiantes a se encaminharem à 
DEAM. Além de proporcionar visibilidade à violência de gênero como questão social, de segurança, justiça e saúde pública.

A Secretária de Desenvolvimento Social - Ana Clara Moreira iniciou sua gestão municipal no ano de 2008. Ana Clara afirmou que "o Conselho Municipal dos Direitos da Mulher em parceria com o Governo do Estado, com a Secretaria Nacional de Políticas para as Mulheres e com a Secretaria Municipal de Desenvolvimento Social, implementou três das principais ferramentas para a garantia dos direitos das mulheres: DEAM, Centro de Referência da Mulher - CRM e a Operação Ronda Maria da Penha (destaca-se que o município de Paulo Afonso - BA foi o terceiro município do interior da Bahia a implantar a referida operação).”

Os Centros de Referência de Atendimento às Mulheres prestam acolhida, acompanhamento psicossocial e orientação jurídica às mulheres em situação de violência (violência doméstica e familiar contra a mulher - sexual, patrimonial, moral, física, psicológica; tráfico de mulheres, assédio sexual; assédio moral; etc) (SECRETARIA POLÍTICAS PARA AS MULHERES, 2017).

O objetivo da Operação Ronda Maria da Penha é atender mulheres vítimas de violência sob medida protetiva instaurada. (SECRETARIA ESTADUAL DE POLITICAS PARA AS MULHERES)

A atual presidente do Conselho Municipal dos Direitos da Mulher - CMDM Izabela Teixeira iniciou sua gestão em 2016 e enfatizou que "a política para a defesa dos direitos das mulheres pauloafonsinas está consolidada e o CMDM tem papel basilar nesta conquista. Temos muitos parceiros, sendo os principais a Secretaria de Desenvolvimento Social e a Delegacia Especializada de Atendimento à Mulher - DEAM".

As ações do CMDM são planejadas mensalmente de acordo com as demandas das reuniões, salientando que devido ao aumento da demanda foi instituída, através de Resolução publicada no Diário Oficial do Município, comissão de monitoramento e fiscalização para os hospitais municipais de modo a garantir os direitos das mulheres, sobretudo, que o atendimento seja humanizado e que as gestantes tenham a presença de um acompanhante durante o parto, afirmou Izabela Teixeira.

Foi constatado que o Conselho Municipal dos Direitos da Mulher realiza constantes ações afim de amenizar o desgaste emocional e garantir os direitos das mulheres que estão no Conjunto Penal Municipal. 
Ressalta-se que devido à complexidade, ao aumento da demanda e visando, principalmente, a garantia dos direitos das mulheres pauloafonsinas o CMDM solicitou ao governador da Bahia: funcionamento 24 horas da DEAM; mais uma delegada titular para DEAM e a implantação da Sala Lilás (espaço designado para oferecer atendimento humanizado às mulheres vítimas de violência física e sexual - sobretudo no exame de corpo de delito) no Instituto Médico Legal (IML).

No que tange ao Composto de Marketing ou Marketing Mix foi observado que o Conselho Municipal dos Direitos da Mulher - CMDM tem como Produto fundamental a promoção, a defesa dos direitos e o empoderamento das mulheres.

Devido o CMDM ser um órgão restrito ao município, a Praça pode ser compreendida como o território de Paulo Afonso - BA. Contudo, municípios circunvizinhos também são contemplados com benefícios alcançados pelo CMDM. A exemplo da DEAM que, apesar de ser para atendimento exclusivo do município de Paulo Afonso, realiza algumas orientações às mulheres de municípios circunvizinhos que são carentes de uma Delegacia Especializada.

A Promoção é realizada através de campanhas educativas, ações continuadas no conjunto penitenciário feminino, visitas e intervenções nos Hospitais Municipais, realizações de Conferências e, sobretudo, constante participação em eventos realizando a defesa e a promoção dos direitos das mulheres. Concomitantemente evidenciando que, por meio do esforço em conjunto, o município de Paulo Afonso - BA atualmente possui implantada uma Delegacia Especializada de Atendimento à Mulher, um Centro de Referência da Mulher - CRM e o Programa Ronda Maria da Penha.

No campo do marketing social, alguns aspectos relacionados ao Preço foram utilizados, como: reconhecimento público, com entrega de placa de agradecimento, pelos relevantes serviços prestados ao Conselho Municipal dos Direitos da Mulher às conselheiras que militaram para implementação do CMDM no município: Marileide Brasil e Edvalda Aroucha. Bem como reconhecimento público, através de entrega de placa de agradecimento aos serviços prestados na defesa e garantia dos direitos das mulheres pauloafonsinas, às delegadas Lígia Nunes, Mirela Santana e a Secretária de Desenvolvimento Social Ana Clara Moreira.

Evidencia-se que o Público-adotante são as mulheres em situação de violência familiar (as que estão na iminência de sofrer; as que estão sofrendo e as mulheres que já sofreram).

No que tange às $\underline{\text { Pessoas, }}$ estas são as pessoas que compõe a rede de defesa dos direitos das mulheres de Paulo Afonso - BA, que, através de ações do Conselho Municipal dos Direitos 
da Mulher - CMDM, foi comprovada ser bem articulada e eficaz no combate a violência doméstica e intrafamiliar.

Foi evidenciado por Mirela Santana que o município necessita da implantação de uma Defensoria Pública Especializada em violência doméstica, bem como de uma Vara Especializada em Violência Doméstica e Familiar, além da ampliação da equipe da Delegacia da Mulher, visto que a mesma também atende crianças, adolescentes e idosos de qualquer sexo.

Evidenciou-se ainda que todas as delegadas entrevistadas sugeriram a implantação de um Abrigo Institucional para as Mulheres pois este subsidiaria completamente a política para a defesa dos direitos das mulheres oferecendo acolhimento temporário para mulheres adultas (com seus filhos), que tenham sofrido violência doméstica, sexual, psicológica ou moral, que necessitam se afastar da residência por estarem sofrendo ameaças e correndo risco de morte. As mulheres poderiam permanecer até conseguirem um lar seguro, fechando assim o ciclo de proteção.

O Abrigo Institucional para Mulheres tem como objetivo a proteção física e emocional da mulher e seus dependentes, a articulação com a rede de serviços da assistência social e do Sistema de Justiça, a superação da situação de violência vivida por meio do resgate da autonomia dessas mulheres e a inclusão produtiva no mercado de trabalho (MINISTÉRIO DE DEFESA SOCIAL).

As atividades do CMDM, tendo como referência o marketing social, compreendem uma conjunção de ações que envolvem, principalmente, campanhas, palestras e cursos. Mas, estas só funcionam, pois estão conectadas a diversas iniciativas que geram mudanças cognitivas, de ação, de conduta e de estima.

Considerando que a análise e realização das ações acima citadas necessitam da aprovação e do subsídio do Governo Estadual e Municipal, as sugestões acima citadas serão encaminhadas ao governador de Estado e a Secretaria de Desenvolvimento Social para análise e, considerando o grau de importância e o comprometimento dos órgãos acima citados para com a defesa dos direitos das mulheres pauloafonsinas, possivelmente serão implementadas. 


\section{Considerações Finais}

Considerando o constante crescimento da violência contra mulher, problema este que assola a sociedade desde a sua origem, o objetivo deste artigo foi analisar, sob a ótica do Marketing Social, a contribuição das ações promovidas pelo Conselho Municipal dos Direitos da Mulher - CMDM em sua missão de garantir os direitos das Mulheres de Paulo Afonso - BA.

Mediante levantamento das ações promovidas pelo Conselho Municipal dos Direitos da Mulher bem como a percepção dos órgãos que também trabalham com a promoção dos direitos da Mulher (Delegacia Especializada no Atendimento à Mulher - DEAM e Secretaria de Desenvolvimento Social - SEDES) foi corroborado, sob a ótica do Marketing Social, que o CMDM tem papel fundamental na política pública municipal, sobretudo na garantia e promoção dos direitos das mulheres pauloafonsinas.

Posteriormente foi verificado que a rede de atendimento às mulheres pauloafonsinas é consolidada e que a parceria com a Secretaria de Desenvolvimento Social - SEDES, a Delegacia Especializada no Atendimento à Mulher - DEAM e a $18^{\mathrm{a}}$ Coordenadoria Regional de Polícia do Interior - COORPIN é fundamental para este fortalecimento.

Analisando, sob a ótica do Marketing Social, foi verificado que, o Conselho Municipal dos Direitos da Mulher - CMDM em parceria Governo do Estado e com a Secretaria de Desenvolvimento Social, evidenciando a promoção e a defesa dos direitos, transformou indubitavelmente a política voltada para as mulheres deste município.

A implementação, sobretudo, de uma Delegacia Especializada no Atendimento à Mulher - DEAM; do Programa Ronda Maria da Penha e do Centro de Referência da MulherCRM revolucionou o panorama municipal atendendo, indiscriminadamente, as necessidades mais complexas da sociedade pauloafonsina e beneficiando indiretamente cidades circunvizinhas.

Através de participações em eventos e reuniões, recepção de denúncias e visitas a órgãos governamentais e não governamentais, o Conselho Municipal dos Direitos da Mulher - CMDM compreende as necessidades e os anseios das mulheres pauloafonsinas e se empenha para que a defesa e a garantia dos direitos das mulheres sejam efetivadas com maior comprometimento e celeridade. 
Sugere-se a realização de futuras pesquisas para avaliar a continuidade do empenho do Conselho Municipal dos Direitos da Mulher - CMDM e dos órgãos responsáveis para com a defesa e garantia dos direitos das mulheres pauloafonsinas bem como a implementação das sugestões aqui citadas.

\section{Referências}

BAHA, Secretaria de Políticas Para as Mulheres - SPM. Ronda Maria da Penha chega ao município de Paulo Afonso. Disponível em http://www.mulheres.ba. gov.br/2016/05/1117/Ronda-Maria-da-Penha-chega-ao-municipio-de-Paulo-Afonso.html. Acesso em 14 de outubro de 2017.

BOONE, Louis E. \& KURTZ, David L. Marketing Contemporâneo, Rio de Janeiro: Editora LTC, 8 ed., 1998.

BRASIL, Secretaria de Defesa Social. Serviço de Acolhimento para Mulheres em Situação de Violência. Disponível em: http://mds.gov.br/assuntos/assistencia-social/unidades-deatendimento/unidades-de-acolhimento/servico-de-acolhimento-para-mulheres-em-situacaode-violencia. Acesso em 06 de abril de 2018.

BRASIL, Secretaria Especial de Políticas para as Mulheres. O que é Delegacia Especializada no Atendimento à Mulher (DEAM)? Disponível em http://www.spm.gov.br/arquivosdiversos/acesso-a-informacao/perguntas-frequentes/violencia/o-que-e-delegaciaespecializada-no-atendimento-a-mulher-deam. Acesso em 14 de outubro de 2017.

BRASIL, Secretaria Especial de Políticas para as Mulheres. O que é Centro de Referência de Atendimento à Mulher em Situação de Violência?. Disponível em: http://www.spm.gov.br/arquivos-diversos/acesso-a-informacao/perguntasfrequentes/violencia/o-que-e-centro-de-referencia-de-atendimento-a-mulher-em-situacao-deviolencia. Acesso em 19 de março de 2018.

BRASIL, Secretaria de Políticas para as Mulheres. Políticas Públicas para as Mulheres. Acesso em 20 de março de 2018 Disponível em: http://www.spm.gov.br/central-deconteudos/publicacoes/publicacoes/2012/politicas_publicas_mulheres.

FONTES, Miguel. Marketing social revisitado: novos paradigmas do mercado social. Florianópolis: Cidade Futura, 2001.

FOX, Karen F. A.; KOTLER, Philip. The Marketing of Social Causes: The First 10 Years. Journal of Marketing, v. 44, Fall 1980, p. 24-33. 
GADE, Christiane. Psicologia do consumidor e da propaganda. Ed. rev. e ampl. São Paulo: EPU, 1998.

GIL, Antônio Carlos. Gestão de Pessoas: enfoque nos papeis profissionais. São Paulo: Atlas, 2001

KOTLER, Philip. Marketing de A a Z: 80 conceitos que todo profissional precisa saber. Rio de Janeiro: Campus, 2003.

;ZALTMAN, G. Social Marketing. Journal of Marketing, 35 (3): 3-12, Summer 1971.

; LEE, Nancy. Up and out of poverty - the social marketing. New Jersey: Wharton School Publishing, 2009.

; ROBERTO, E. L. Marketing social: estratégias para a mudança de comportamento do público. New York, NY: The Free Press, 1989.

LAKATOS, Eva Maria \& MARCONI, Maria de Andrade. Fundamentos de Metodologia Científica/-4.ed.Ver.E ampl. - São Paulo: Atlas 2001.

MATTAR, Fauzer Najib. Pesquisa de Marketing: metodologia, planejamento. 5 ed. São Paulo: Atlas, 1999.

SILVA, Maria Gabriela Jandiroba. Marketing Social interno relacionado a questões ambientais: uma investigação numa instituição federal de ensino superior. Dissertação (Dissertação em Administração). Escola de Administração da Universidade Federal da Bahia. Salvador - BA. 2016.

VAZ, G. N. Marketing institucional: um mercado de ideias. São Paulo: Pioneira, 1995.

YIN, Robert K. Estudo de caso: planejamento e métodos / Robert K. Yin; trad. Daniel Grassi - 2.ed. -Porto Alegre : Bookman, 2001. 1.

Como citar este artigo (Formato ABNT):

FRANCHINI , Aline Bezerra; SILVA, Bruno Diniz G. da; SILVA, Maria Gabriela J. Marketing Social e a Promoção dos Direitos da Mulher: um Estudo de Caso. Id on Line Revista Multidisciplinar e de Psicologia, 2018, vol.12, n.41, p.1-14. ISSN: 1981-1179. 